\title{
EL PROCESO DE INSTITUCIONALIZACIÓN DE LAS RELACIONES EUROMEDITERRÁNEAS Y EL ÁMBITO DE LOS INTERCAMBIOS COMERCIALES AGRÍCOLAS: LA PRIMACÍA DE LOS OBJETIVOS DE SEGURIDAD
}

\author{
María Ángeles Alaminos ${ }^{58}$ \\ Universidad Complutense de Madrid (UCM) / UNISCI
}

\begin{abstract}
Resumen:
En este artículo analizamos, desde una óptica centrada en Europa, la evolución de las relaciones en la región mediterránea desde los primeros intentos de las comunidades europeas de formalización de acuerdos comerciales y desde los primeros pasos hacia la institucionalización de las políticas mediterráneas. En el ámbito del comercio de productos agrícolas argumentamos que la formulación de las diferentes iniciativas depende de criterios políticos y securitarios europeos. La política en materia de comercio de alimentos con los países vecinos de la orilla sur del Mediterráneo no se formula desde preocupaciones ligadas a la seguridad alimentaria ni al desarrollo económico de dichos países, sino que entran en juego intereses más conservadores en el ámbito de la seguridad y de la estabilidad, así como de la agenda normativa de la Unión Europea.
\end{abstract}

Palabras clave: Relaciones euro-mediterráneas, Asociación Euro-Mediterránea, Política Europea de Vecindad, zona de libre comercio, intercambios comerciales agrícolas, seguridad.

Title in English: "The Process of Institutionalization in Euro-Mediterranean Relations and Trade Exchanges in the Agrarian Sector: The Focus on Security”.

\begin{abstract}
:
In this article the evolution of North South relations in the Mediterranean region is analyzed from a European perspective. It starts with the early attempts of the European Communities to negotiate trade agreements, and the first steps in the institutionalization of Mediterranean policies. In the area of trade on agricultural products it is argued that the policy formulation depends on the European political and security criteria. Food trade policy with the neighboring countries on the southern shore of the Mediterranean is not developed taken into consideration concerns related to food security and economic development in these countries. On the contrary it is subject to constraints related to European interests in security and stability, as well as the promotion of the EU normative agenda.
\end{abstract}

Keywords: Euro-Mediterranean relations, Euro-Mediterranean Partnership, European Neighborhood Policy, free trade area, agricultural trade, security.

Copyright (C) UNISCI, 2013.

Las opiniones expresadas en estos artículos son propias de sus autores, y no reflejan necesariamente la opinión de UNISCI. The views expressed in these articles are those of the authors, and do not necessarily reflect the views of UNISCI.

\footnotetext{
${ }^{58}$ María Ángeles Alaminos Hervás es becaria de investigación en la Universidad Complutense de Madrid, investigadora junior de UNISCI y miembro del Foro Hispano-Argelino.

Dirección: Departamento de Estudios Internacionales, Facultad de Ciencias Políticas y Sociología, UCM, Campus de Somosaguas, 28223 Madrid, España.

E-mail: ma_alaminos_h@hotmail.com.

http://dx.doi.org/10.5209/rev_UNIS.2013.n31.4470
} 


\section{Introducción}

En este artículo trataremos la evolución de las relaciones de la Unión Europea (UE) con los países del Magreb - Marruecos, Túnez y Argelia - dentro del marco amplio de las relaciones euro-mediterráneas, y concentraremos nuestro interés en el comercio de productos agrícolas entre las dos riberas del Mediterráneo.

No abordamos en este artículo el periodo de la historia colonial, que marca profundamente las relaciones de los países europeos con sus vecinos del norte de África, en particular el legado colonial francés y su influencia respecto a los tres países del Magreb en los que profundizamos en este texto.

Ello no obsta para constatar como premisa previa que el periodo colonial marcó - y sigue influenciando - las relaciones euro-mediterráneas, generando patrones de dependencia y asimetría entre las dos orillas del Mediterráneo. Algunos autores sostienen en este sentido que "in the European process for the Mediterranean, the European Union proposes and frequently orders. The South, that is to say, essentially the 'Arab world', divided and disunited, reacts on most occasions in a fragmented way, without really influencing the course of a process directed from Brussels. ${ }^{59}$

Analizaremos, desde una óptica centrada en Europa - en cuanto a la visión estratégica europea, los intereses y los acontecimientos concernientes a la integración interna de la UE que han influenciado y condicionado la formulación y revisión de las políticas euromediterráneas - la evolución de las relaciones en la región mediterránea desde los primeros intentos de las comunidades europeas de formalización de acuerdos comerciales y desde los primeros pasos hacia la institucionalización de las políticas mediterráneas.

En este sentido, debemos resaltar también que nuestra zona de interés, el Magreb (Marruecos, Túnez y Argelia), se define y se manifiesta como una zona heterogénea a nivel político y económico, caracterizada por su débil integración interna y su dependencia del mercado europeo lo cual dificulta por un lado su definición como 'región' y por otro lado la posibilidad de establecer relaciones con la Unión Europea como un grupo homogéneo. En consecuencia, estudiaremos tanto los marcos políticos y económicos de las relaciones a nivel multilateral entre la UE y sus socios mediterráneos como la prioridad otorgada a las relaciones bilaterales.

La hipótesis inicial que guía el enfoque y el desarrollo de este texto plantea que las políticas de importación y exportación de alimentos, en el caso de la Política Europea de Vecindad, y en el marco más amplio de la evolución de la política euro-mediterránea, dependen de criterios políticos y securitarios europeos. La política en materia de comercio de alimentos con los países vecinos de la orilla sur del Mediterráneo no se formula desde preocupaciones ligadas a la seguridad alimentaria ni al desarrollo económico de dichos países, sino que entran en juego intereses más conservadores en el ámbito de la seguridad y de la estabilidad, así como de la agenda normativa de la Unión Europea.

Abordaremos en primer lugar los orígenes y la evolución de la política europea hacia el Mediterráneo desde la década de los años 60, incidiendo en los aspectos políticos y económicos - con énfasis en los intercambios agrícolas - fundamentales para la comprensión

\footnotetext{
${ }^{59}$ Zaim, Fouad (1999): "The third generation of Euro-Mediterranean association agreements: A view from the south”, Mediterranean Politics, vol.4, n² (Summer 1999), pp.36-37.
} 
de las relaciones contemporáneas entre la UE y sus socios mediterráneos. En el progresivo proceso de institucionalización de las políticas europeas podremos observar algunos de los patrones que aún hoy marcan las relaciones euro-mediterráneas, a pesar de los continuos esfuerzos de reflexión y revisión de los distintos marcos institucionales establecidos y de las distintas políticas formuladas.

En un segundo lugar analizaremos los cambios fundamentales en las relaciones euromediterráneas acontecidos con el final de la Guerra Fría. El cambio fundamental de las relaciones euro-mediterráneas se dio con el establecimiento de una Asociación EuroMediterránea (AEM). La idea, esbozada en 1992, vio la luz en el Consejo Europeo de Corfú, celebrado en junio de 1994 y se formalizó institucionalmente con la Declaración de Barcelona en noviembre de 1995. El balance de la AEM, y su sucesor institucional en 2008, la Unión por el Mediterráneo, deja una visión bastante negativa del proceso; veremos las causas de este fracaso y las consecuencias para el sector agrícola de los países caso de estudio.

En tercer lugar estudiaremos el marco político más novedoso de las relaciones de la UE con el Mediterráneo; nos referimos a la Política Europea de Vecindad (PEV). En este epígrafe abordaremos los objetivos y principios de la PEV, analizando los aspectos más novedosos del nuevo marco político, así como las limitaciones y ambigüedades de que adolece tal aproximación, enfatizando los aspectos securitarios y normativos y la tensión con los objetivos formalmente declarados.

Por último expondremos brevemente, en función del enfoque diferenciado preconizado por la PEV, la diversa forma en que se han visto afectadas las relaciones de la UE con Marruecos, Túnez y Argelia, para terminar de forma concisa con algunas conclusiones preliminares derivadas de este análisis.

\section{Primeros pasos en el proceso de institucionalización de la política europea hacia el Mediterráneo}

Las relaciones de la Unión Europea (UE) con los países de la ribera sur del Mediterráneo se remontan a la década de los años 60. Poco después de la entrada en vigor de los Tratados de Roma en 1958, en los que encontramos las primeras alusiones a Túnez y Marruecos, la Comunidad Económica Europea (CEE), influenciada por las relaciones de Francia con los países del Magreb, comienza las negociaciones de los primeros acuerdos comerciales con Marruecos y Túnez, los cuales se firman en $1969 .{ }^{60}$

\subsection{La Política Mediterránea Global}

En la cumbre de París de 1972, se acuerda la institucionalización de las relaciones, fundamentalmente comerciales, con los países del Mediterráneo a través de la denominada 'Política Mediterránea Global' o 'Enfoque Global de las relaciones entre las Comunidades europeas y los países del Mediterráneo’, vigente durante el periodo 1976-1990:

\footnotetext{
60 Como hicimos explícito en la introducción, no abordaremos en este artículo el periodo histórico de las relaciones coloniales de algunos de los países europeos con el norte de África, particularmente Francia, España, Italia, pero consideramos - como premisa previa - que su influencia es determinante en la naturaleza de las relaciones y en el establecimiento de los progresivos marcos políticos y económicos en el ámbito euromediterráneo.
} 
"en vue d'une approche globale des relations entre les Communautés européennes et les pays du Bassin méditerranéen, il s'agissait à la fois de confirmer la politique menée jusqu'ici par les Communautés et de définir leur politique future". ${ }^{61}$

Esta iniciativa supuso, por un lado, la 'invención del Mediterráneo', es decir, la 'delimitación del campo de aplicación territorial' de la política y por otro lado, el 'enfoque global' buscó dar respuesta a la situación ocasionada por la primera ampliación de la Comunidad, intentando adaptar y aglutinar los diferentes acuerdos concertados de forma ad hoc hasta aquel momento, sistematizando de alguna manera las relaciones de la CEE con los países del Magreb y más generalmente, con los países del Mediterráneo. ${ }^{62}$

La consecuencia inmediata de la 'Política Mediterránea Global' fue la negociación de nuevos acuerdos - 'acuerdos de asociación de primera generación' - con algunos países del Mashrek y del Magreb, incluyendo el establecimiento de acuerdos de cooperación con los países objeto de nuestro interés particular en este texto, Marruecos, Argelia y Túnez en 1976, así como la firma de protocolos de cooperación técnica y financiera.

El 'enfoque global' mostró no obstante sus limitaciones ante la expectativa de ampliación de las Comunidades Europeas a tres países del sur de Europa - realizándose la adhesión formal de Grecia en 1981, y de España y Portugal en 1986 - por el impacto de su producción agrícola en el mercado común y en las relaciones con terceros países, fundamentalmente en lo concerniente con la región mediterránea.

En junio de 1979, la Comisión presentó un informe al Consejo en el que se refería a esta situación con denominaciones como 'the invasion of Mediterranean produce':

“Enlargement will increase the Community's GDP by only a tenth, and its population by a mere fifth, but agricultural output will rise by a quarter and the numbers employed in agriculture, and the area of farmland, by about half". ${ }^{63}$

Añadiendo más adelante:

"There will be problems, and they will focus mainly on the Mediterranean region, for while the accession of three southern European countries will shift the EEC's centre of gravity more towards the Mediterranean, it could, if care is not taken, give rise to a certain amount of friction with the other countries in the area, countries which might find it very hard to understand why, when some of them have long had special relations with Europe, enlargement should take place at their expense". ${ }^{4}$

${ }^{61}$ Commission des Communautés Européennes (1972) : 'Communication de la Commission au Conseil', 27 Septiembre 1972, SEC (72) 3111 final, p. 1.

${ }^{62}$ Commission des Communautés Européennes (1972), op. cit., p.5 y Bicchi, Federica(2010): 'The Impact of the ENP on EU-North Africa Relations: The Good, the Bad and the Ugly', en Whitman, Richard G. y Wolff, Stefan (eds.): The European Neighbourhood Policy in Perspective: Context, Implementation and Impact, Basingstoke, Palgrave Macmillan, p. 207.

${ }^{63}$ Commission of the European Communities (1979): "The Second Enlargement of the European Community", Luxembourg: Office for Official Publications of the European Communities, p.15.

${ }^{64}$ Commission of the European Communities (1979), op. cit., p. 22. 
En el documento de 1979, la Comisión especificaba las futuras consecuencias políticas, económicas y sociales de la segunda ampliación respecto a la situación y las relaciones con los países no miembros de la ribera sur y este del Mediterráneo, incidiendo en la posible pérdida de mercado europeo para un número importante de las exportaciones de productos agrícolas procedentes de dichos países: ${ }^{65}$

"Let us take a couple of specific items: $96 \%$ of Moroccan exports of tomatoes and $87 \%$ of Tunisian exports of olive oil are sent to the EEC. Once the EEC has been enlarged to twelve it will be nearer to self-sufficiency in these products and purchases will consequently be cut. The EEC will be almost self-sufficient in olive oil and will have a wine surplus. After enlargement it will be able to cover $76 \%$ of its citrus fruit needs (compared with $41 \%$ before) and $85 \%$ of fresh fruit demand (compared with $78 \%$ before). For the other Mediterranean countries, however, agriculture is still a vitally important part of their economies which in Algeria, Morocco, Tunisia, Egypt and Syria occupies between $45 \%$ and $55 \%$ of the labour force". ${ }^{66}$

\title{
2.2. La necesidad de un proceso de revisión
}

En 1982 la Comisión profundizó en la necesidad de reconsiderar la Política Mediterránea de la Comunidad Económica Europea ante las limitaciones del 'enfoque global' y destacó 'la necesidad de una nueva iniciativa' que tuviera en cuenta el impacto de la segunda ampliación o ampliación hacia el sur. ${ }^{67}$

En el ámbito agrícola, los problemas y limitaciones del enfoque global de 1972 se resumían así:

\begin{abstract}
"The Community's internal Mediterranean problems, due largely to the legacy of the past and exacerbated by a common agricultural policy skewed against Mediterranean crops and an inadequate regional development policy have made it impossible to give practical effect to the review clauses in the various agreements (...) In these circumstances the Mediterranean partners' aggregate trade deficit with the Community, which the agreements had been intended to reduce, actually grew from just over $4000 \mathrm{~m}$ ECU in 1973 to just over $9000 \mathrm{~m}$ ECU in 1979. This trend has greatly contributed to the decision of some of our partners to impose severe import curbs which have hit Community exports hard. As essential food imports, a major item, cannot be cut back, these curbs have fallen disproportionately on capital goods, which in the long run could jeopardize the whole development process". ${ }^{68}$
\end{abstract}

A la inconsistencia de la política mediterránea de la CEE se añadía, como en documentos anteriores, el reto que suponía la ampliación de la Comunidad a países como España y Portugal, con una producción agrícola muy relevante y acceso al mercado común:

"in the short term the export openings created for our Mediterranean partners on the

Portuguese and Spanish markets will be extremely limited, whereas the new Member States will

\footnotetext{
${ }^{65}$ Los países no miembros de la ribera sur y este del Mediterráneo se denominan en inglés 'Mediterranean nonmember countries', de ahí su acrónimo (MNC) utilizado en algunos de los documentos europeos. Otro acrónimo utilizado frecuentemente en los documentos oficiales europeos para nombrar al grupo de países de la ribera sur y este del Mediterráneo es SEM o SEMCs (Southern and Eastern Mediterranean Countries).

${ }^{66}$ Commission of the European Communities (1979), op. cit., p. 23.

67 Commission of the European Communities (1982): "On a Mediterranean Policy for the Enlarged Community", Commission Communication to the Council, 24 Junio 1982, COM (82) 353 final.

${ }^{68}$ Commission of the European Communities (1982), op. cit., pp. 2-3.
} 
immediately get a real competitive advantage on the Community market (...) the agricultural products to be considered in assessing the impact of enlargement were citrus fruits, particularly oranges and small fruits, fresh tomatoes, potatoes, especially new potatoes, wine and olive oil, which account for the bulk of the Community's agricultural imports from countries in the Mediterranean area. They are also the ones which are posing problems because of the level of Spain's present and potential production". ${ }^{9}$

En el caso de los países que nos interesan de forma particular en este trabajo, Argelia sería el país menos afectado por la perspectiva de la segunda ampliación, dada la naturaleza de sus exportaciones, principalmente petróleo y sus derivados, con lo que no se vería afectado por la competencia española y portuguesa tras la accesión de dichos países a la Comunidad Europea en 1986. Sin embargo, en el caso de Marruecos y Túnez, mucho más dependientes del mercado comunitario y exportadores de productos 'sensibles', el impacto de la segunda ampliación se atisbaba a priori de forma muy negativa:

\begin{abstract}
“Morocco's exports to the Community represent a large part of its GNP, and despite its substantial phosphate exports (about $25 \%$ of total exports)it is still heavily dependent on sales of Mediterranean fruit and vegetables (over 50\% of total agricultural exports) and of textile products (15\%). Tunisia is likewise very dependent on a number of highly sensitive products, particularly olive oil, citrus fruits and textiles. Olive oil in particular not only accounts for more than 50\% of the country's agricultural exports and more than $10 \%$ of its total exports, but is a major source of employment (olive oil is the exclusive or main source of income of about $20 \%$ of the population)". ${ }^{70}$
\end{abstract}

De forma general tenemos además que tener en cuenta, por un lado, que los cambios internos derivados del avance del proyecto europeo, con la segunda ampliación hacia el sur y el correspondiente proceso de integración de los nuevos Estados miembros - Grecia, España y Portugal - durante la década de los ochenta ocupó gran parte del interés y de la agenda de la Comunidad Europea, marginando de cierta manera las relaciones con terceros países de la cuenca mediterránea. Por otro lado, la adhesión de dichos países proporcionó a la Comunidad una mayor auto-suficiencia alimentaria (y en otros sectores) respecto a ciertos productos antes importados de los países mediterráneos no miembros (dependientes del mercado europeo en las exportaciones de esos productos 'sensibles'), pero también acercó geográficamente a la Comunidad al espacio mediterráneo, cambiando en cierta forma el centro de gravedad europeo y generando mayores relaciones de interdependencia entre las dos orillas del Mediterráneo y preocupación en cuanto a situaciones de inestabilidad que pudieran afectar a la seguridad de Europa y a su abastecimiento energético. ${ }^{71}$ La accesión de Grecia, España y Portugal a las Comunidades Europeas fue también un factor de reforzamiento de las relaciones políticas de Europa con el Mediterráneo. Estos países, junto con Francia e Italia,

\footnotetext{
${ }^{69}$ Ibid., pp. 4-5.

${ }^{70}$ Ibid., p. 7.

${ }^{71}$ Colombo, Silvia y Abdelkhaliq, Nur: "The European Union and Multilateralism in the Mediterranean: Energy and Migration Policy", Mercury, e-paper nº (January 2012), p. 7, en http://www.mercuryfp7.net/fileadmin/user_upload/E-paper_no18 Jan2012.pdf.
} 
constituyeron una especie de "southern club within the EU that has acted as a lobby in favor of the Mediterranean in terms of policy initiatives and resource allocations". ${ }^{72}$

\subsection{Una "Nueva Política Mediterránea"}

Tras el proceso de revisión de la política mediterránea de la Comunidad Europea, la Comisión propuso en 1990 una nueva reorientación de la política mediterránea para el periodo 1992-96, una 'Nueva Política Mediterránea' (NPM) o 'estrategia Mediterránea' renovada que fortaleciera las relaciones exteriores con los países mediterráneos no miembros. ${ }^{73}$

En el documento de la Comisión - 'Redirecting the Community's Mediterranean Policy'- encontramos algunos elementos fundamentales de naturaleza normativa y de seguridad que definen la dimensión exterior de la política europea hacia el Mediterráneo y que se van a manifestar de forma constante en posteriores documentos y de forma más o menos explícita en el desarrollo de progresivas iniciativas y políticas europeas.

Podemos destacar el aspecto securitario, que va a influenciar e impregnar de forma creciente la formulación de políticas posteriores hacia la región, particularmente con el fin de la Guerra Fría, como veremos más adelante. El Mediterráneo se describe como una posible fuente de inestabilidad que puede afectar a la seguridad de Europa, y la dimensión securitaria se comienza a manifestar en la retórica europea en relación íntima con aspectos de desarrollo económico y social:

“(...) owing to geographical proximity and the closeness of all types of relations, stability and prosperity in the Mediterranean non-member countries are key factors in the stability and prosperity of the Community itself. If the courses followed by the Community and the MNC caused a worsening of the economic and social disparities between the two, the Community would be in a difficult position. What is at issue is its security in the broadest sense" ${ }^{74}$

La nueva estrategia mediterránea refleja también algunos elementos normativos prácticamente omnipresentes en la retórica europea y constantes en la formulación de sus políticas. En aras de sus 'responsabilidades en esa parte del mundo', es el 'deber' de la Comunidad el de apoyar la liberalización económica y fomentar la democratización pluralista. Este 'deber' de la Comunidad tiene además como objetivo la convergencia y el acercamiento de los países del Mediterráneo (no miembros) con la Comunidad, mediante la aceptación de los valores que están en la base de la construcción europea:

"The political moves towards pluralism, democratic political procedures and the respect of human rights under way in a number of the MNC are essential to their development and to the creation of a rapprochement with the Community". ${ }^{75}$

\footnotetext{
72 Tanner, Fred: "North Africa: partnership, exceptionalism and neglect", en Dannreuther, Roland (ed.) (2004): European Union Foreign and Security Policy: Towards a neighbourhood strategy, London, Routledge, pp.136137.

${ }^{73}$ Commission of the European Communities (1990): "Redirecting the Community's Mediterranean Policy: Proposals for the period 1992-96", Communication from the Commission to the Council, 1 Junio 1990, SEC (90) 812 final.

${ }^{74}$ Commission of the European Communities (1990), op. cit., p. 2.

${ }^{75}$ Ibid., pp. 2-3; (énfasis en el original).
} 
Como la tercera generación de protocolos financieros con los países del Sur y del Este del Mediterráneo tenía como fecha de expiración octubre de 1991, la nueva política mediterránea renovó los protocolos para cubrir el periodo 1992-1996 y aumentó de forma sustancial el presupuesto dedicado a ellos. $^{76}$

En el ámbito agrícola, tenemos que destacar que una de las cuatro prioridades sectoriales identificadas por la Comisión fue la de reducir la dependencia alimentaria en la región y trabajar en pos de la autosuficiencia alimentaria, buscando la diversificación y promoviendo la producción alimentaria de los países no miembros:

"The Commission considers it essential that the bulk of financial resources should be allocated to projects designed to achieve greater food self-sufficiency. Even more than in the past, the Commission will see to it that counterpart funds from food aid are used in close conjunction with projects financed under Financial Protocols". 77

Sin embargo, a pesar de las nuevas iniciativas y del aumento considerable de los fondos atribuidos bajo la Nueva Política Mediterránea, ésta no fue capaz de generar los resultados esperados. En el ámbito agrícola, la situación de dependencia en cuanto a las exportaciones agrícolas provenientes del mercado europeo prosiguió y no se consiguió reforzar de forma considerable la 'cooperación horizontal' con el grupo de países implicados de la ribera sur y este del Mediterráneo. En un informe sobre el progreso en la implementación de los proyectos financiados en el Mediterráneo, tanto a nivel bilateral como multilateral, y en una Comunicación de la Comisión al Consejo y al Parlamento Europeo se refleja esta realidad:

\begin{abstract}
"Access to the Community market for agricultural products has substantially improved over the past two decades. There has been a gradual elimination of customs duties on major agricultural products from the MNC (this has been within the bounds of tariff quotas in the case of sensitive products). Even considering the presence of quotas, the products have been admitted at preferential rates of duty. (...) Viewed against the background of the MNCs' own imports, trade in agricultural products demonstrates the failure of efforts at diversification, since in the case of most of the countries self-sufficiency in food commodities has not increased. Dependence on imports from the Community remains the rule". ${ }^{78}$
\end{abstract}

\footnotetext{
${ }^{76} \mathrm{El}$ incremento programado en el documento refleja las siguientes cifras:

"a five-year (1992-1996) allocation of ECU 1955 million as compulsory expenditure to provide support for this policy (current prices) (...) an indicative five-year (1992-1996) allocation of ECU 480 million as noncompulsory expenditure (1991 prices) (...) call on the EIB to make large-scale use of non-protocol financing in the MNC and, to this end, set a ceiling of ECU 3.5 billion for the period 1992-96". Commission of the European Communities (1990), op. cit., p.18; En nuestros países casos de estudio, los fondos atribuidos constituyeron un aumento considerable respecto a los fondos anteriores: "Algeria's overall allocation increased by $46 \%$ against the previous protocol, the biggest percentage rise. EIB loans increased by 53\% from ECU 183 million to ECU 280 million, while the budget fund allocation rose by $25 \%$ ". En el caso de Marruecos "the overall package of ECU 438 million is up $35 \%$ on the third protocol total, with the budget fund allocation rising $26 \%$ and EIB loans 46\%". Y Túnez "will receive a total of ECU 284 million under the fourth protocol, consisting of ECU 116 million in budget funds (including ECU 15 million in risk capital) and ECU 168 million in the form of EIB loans. This represents a $27 \%$ increase on the third protocol", en: Commission of the European Communities (1994a): "Report from the Commission to the Council and the European Parliament on the implementation of financial and technical cooperation with Mediterranean non-member countries and on financial cooperation with those countries as a group", 18 November 1994, COM (94) 384 final, pp. 11, 14 y 15.

${ }^{77}$ Commission of the European Communities (1990), op. cit., p. 14.

${ }^{78}$ Commission of the European Communities (1994a): "Report from the Commission to the Council and the European Parliament on the implementation of financial and technical cooperation with Mediterranean nonmember countries and on financial cooperation with those countries as a group", 18 November 1994, COM (94)
} 


\section{Un cambio fundamental en las relaciones euro-mediterráneas: la Asociación Euro-Mediterránea o Proceso de Barcelona}

El final de la Guerra Fría trajo consigo cambios sustanciales en las relaciones euromediterráneas. De forma general, el fin de la Guerra Fría permitió el reforzamiento de la posición internacional de la Comunidad Europea, algo que quedó de manifiesto con la firma del Tratado de la Unión Europea en Maastricht el 7 de febrero de 1992 - ratificado el 1 de enero de 1993 - origen de la creación de la Unión Europea formada por tres pilares: las Comunidades Europeas, la política exterior y de seguridad común (PESC) y la cooperación policial y judicial en materia penal. En nuestro caso específico, el fin de las divisiones ideológicas permitió un mayor acercamiento político hacia la región mediterránea en su globalidad. $^{79}$

Durante el Consejo Europeo de Lisboa en junio de 1992, la seguridad y estabilidad en el Mediterráneo se consideró como un asunto de fundamental importancia para la Unión Europea:

"The southern and eastern shores of the Mediterranean as well as the Middle East are geographical areas in relation to which the Union has strong interests both in terms of security and social stability". ${ }^{80}$

La región mediterránea quedará retratada como un objetivo clave para la recién nacida PESC y como un posible primer terreno de experimentación de las acciones conjuntas de dicha política. En consecuencia, la región mediterránea se situará bajo la competencia tanto de las relaciones exteriores de la Comunidad o primer pilar, como de la PESC o segundo pilar, y quedará subdividida en dos zonas de interés: el Magreb y Oriente Medio (los países del Mashrek e Israel), objeto de sendos anexos III y IV de las Conclusiones de la Presidencia. ${ }^{81}$

\subsection{El nacimiento de la AEM}

El cambio fundamental de las relaciones euro-mediterráneas se dio con el establecimiento de una Asociación Euromediterránea (AEM). La idea, esbozada en 1992, vio la luz en el Consejo Europeo de Corfú, celebrado en junio de 1994. La evaluación de la política global de la Unión Europea en la región mediterránea llevó a la discusión sobre las necesidades de reforzar la política mediterránea de la UE y desembocó en la búsqueda de posibles iniciativas para reforzar la política tanto a corto plazo como a medio plazo, reflexionando sobre la posibilidad de celebrar una conferencia que contara con la presencia de los países europeos y de los socios mediterráneos. ${ }^{82}$

384 final y Commission of the European Communities (1994b): "Strengthening the Mediterranean policy of the European Union: establishing a Euro-Mediterranean partnership", Communication from the Commission to the Council and the European Parliament, 19 October 1994, COM (94) 427 final, Annex 2, p. 22.

${ }^{79}$ Tanner, op. cit., p. 137.

${ }^{80}$ European Council (1992): "Lisbon European Council", 26-27 June 1992, Bulletin of the European Communities, $n^{\circ}$ 6, p. 20.

${ }^{81}$ European Council (1992), op. cit.; Whitman, Richard G. (2001): "Five years of the EU's Euro-Mediterranean Partnership: progress without partnership?", ISA (Convention Paper Archive), (20-24 February 2001), Chicago; y Tanner, op. cit., p. 137.

${ }^{82}$ European Council (1994a): "Corfu European Council", 24-25 Junio 1994, Bulletin of the European Communities, $n^{\circ}$ 6, p. 13. 
La respuesta de la Comisión a los desafíos planteados por el Consejo Europeo llegó en la Comunicación de octubre de 1994, bajo el título "Strengthening the Mediterranean Policy of the European Union: Establishing a Euro-Mediterranean Partnership". ${ }^{83}$

La Comisión reafirmaba en este documento la importancia estratégica de la cuenca mediterránea y la interdependencia de las dos orillas del Mediterráneo en áreas de vital interés como la energía, la migración, la inversión y el comercio. El objetivo de la Unión Europea era reforzar la política mediterránea estableciendo una asociación euro-mediterránea. La Comisión proponía la creación de un 'espacio económico euro-mediterráneo' caracterizado por el progresivo establecimiento de una zona de libre comercio, una mayor integración económica y una cooperación reforzada en materia política y de seguridad. La asociación euro-mediterránea serviría también para ayudar en la creación de una zona de paz y estabilidad así como en la generación de condiciones de desarrollo económico y social de los países mediterráneos, sobre la base de un diálogo político basado en el respeto de la democracia, el buen gobierno y los derechos humanos. En consecuencia, la Comisión propuso la celebración de una Conferencia Ministerial Euro-Mediterránea en 1995 con el fin de acordar las directrices políticas y económicas a largo plazo que llevaran al establecimiento de un marco institucional duradero que superase las limitaciones de las anteriores políticas mediterráneas de la Comunidad. ${ }^{84}$

Si el Consejo Europeo de Cannes reafirmó la importancia estratégica de las relaciones con los socios mediterráneos y supuso un paso más en el camino hacia el establecimiento de la Asociación Euro-Mediterránea, su aportación fundamental fue la decisión del volumen de las aportaciones para la cooperación financiera con los países de la cuenca mediterránea para el periodo 1995-1999, provenientes del octavo Fondo Europeo de Desarrollo (FED), con un montante total que ascendía a 4685 millones de ECU. ${ }^{85}$

La conferencia ministerial euro-mediterránea se celebró un año más tarde, los días 27 y 28 de noviembre de 1995, en Barcelona, formalizando así la propuesta de la Comisión y logrando el establecimiento de un nuevo tipo de relaciones euro-mediterráneas - dando paso a la Asociación Euro-Mediterránea y sentando las bases del denominado 'Proceso de Barcelona' - basado en la ambiciosa visión estratégica de la UE y en un marco político normativo e integral que cubría toda la región mediterránea, incluyendo los países del sur y del este del Mediterráneo y también algunos socios normalmente considerados geográficamente como situados en Oriente Próximo. ${ }^{86}$ La AEM se concretó en la adopción de la Declaración final de Barcelona por parte de los Estados miembros de la UE y de los doce Terceros Países Mediterráneos (TPM) siguientes: Argelia, Chipre, Egipto, Israel, Jordania, Líbano, Malta, Marruecos, Siria, Túnez, Turquía y la Autoridad Palestina. ${ }^{87}$

\footnotetext{
${ }^{83}$ Commission of the European Communities (1994b): "Strengthening the Mediterranean policy of the European Union: establishing a Euro-Mediterranean partnership", Communication from the Commission to the Council and the European Parliament, 19 Octubre 1994, COM (94) 427 final.

${ }^{84}$ Commission of the European Communities (1994b), op. cit.; y European Council (1994b): "Essen European Council”, 9-10 December 1994, Bulletin of the European Communities, nº 12, pp.7-27.

${ }^{85}$ Los fondos, como veremos más adelante, fueron plasmados en el programa MEDA. European Council (1995): "Cannes European Council", 26-27 June 1995, Bulletin of the European Communities, no 6, pp.9-32.

${ }^{86}$ Zaim, op. cit., pp. 36-37; Tanner, op. cit., p. 137; y Keukeleire, Stephan y MacNaughtan, Jennifer (2008): The Foreign Policy of the European Union, Basingstoke, Palgrave Macmillan, p. 274.

${ }^{87}$ Euro-Mediterranean Conference (1995): Barcelona Declaration, 27-28 November 1995.
} 


\subsection{Las bases del Proceso de Barcelona}

El objetivo general de la AEM era el de establecer un nuevo marco de cooperación multilateral para las relaciones con el Mediterráneo, una 'asociación comprehensiva' que abarcara la totalidad de la región mediterránea con "el objetivo general de hacer de la cuenca mediterránea un ámbito de diálogo, intercambio y cooperación que garantice la paz, la estabilidad y la prosperidad". ${ }^{88}$ La idea de asociación o partenariado constitutía un objetivo que sería alcanzado de forma progresiva, no inmediata, mediante la profundización de las relaciones y el desarrollo paulatino de una zona de libre comercio. ${ }^{89}$

El primer objetivo de la AEM, plasmado en la 'asociación política y de seguridad', es el de favorecer el surgimiento de un 'espacio común de paz y estabilidad' en el Mediterráneo, "en mantener un diálogo político más intenso a intervalos regulares". El capítulo económico y financiero, aspecto predominante de la asociación, aspira al desarrollo socioeconómico sostenible y equilibrado "con el fin de alcanzar su objetivo de crear una zona de prosperidad compartida". Finalmente, en el cesto socio-cultural y humano "los participantes reconocen que las tradiciones de cultura y de civilización de todo el Mediterráneo, el diálogo entre estas culturas y los intercambios humanos, científicos y tecnológicos son un factor esencial para el acercamiento y la comprensión entre sus pueblos y para la mejora de su percepción recíproca.". 90

De esta forma la AEM se manifestaba, al menos formalmente, como una iniciativa destinada por un lado a desarrollar el diálogo político multilateral, complementado por un diálogo bilateral diferenciado establecido con cada uno de los socios mediante los 'acuerdos euro-mediterráneos de asociación'. Por otro lado, el pilar esencial de la AEM era el refuerzo de las relaciones económicas, permitiendo la instauración de una zona de libre comercio que permitiera la eliminación progresiva de los obstáculos aduaneros (arancelarios y no arancelarios) en los intercambios comerciales de productos manufacturados, no así de los productos agrícolas tradicionalmente protegidos ni de los servicios, aunque se prevé hacerlo en un futuro:

"taking as a starting point traditional trade flows, and as far as the various agricultural policies allow and with due respect to the results achieved within the GATT negotiations, trade in agricultural products will be progressively liberalized through reciprocal preferential access among the parties; trade in services including right of establishment will be progressively liberalized having due regard to the GATS agreement". ${ }^{91}$

A este respecto, se estableció un programa de trabajo quinquenal y se creó un nuevo instrumento financiero para la realización de la asociación y de sus actividades: MEDA (Mesures d'Accompagnement), que aportaría considerables fondos para jugar un papel importante en los esfuerzos de reestructuración económica de los socios mediterráneos. La realización progresiva de los acuerdos de asociación requiere:

\footnotetext{
${ }^{88}$ Ibid., p. 2.

${ }^{89}$ Whitman (2001), op.cit.

${ }^{90}$ Euro-Mediterranean Conference, op. cit., pp. 2-6.

${ }^{91}$ Ibid., p. 4.
} 
"That the market access, which the EU had already given to the North Africans, now has to be reciprocated. This is done in a staged manner over 12 years. The tariffs for non-sensitive products are removed over the first three years. Tariffs for products likely to be affected by European competition are removed from year four to 12 and tariffs for the most sensitive products are removed from year six to $12, .{ }^{92}$

De esta manera, los socios de la ribera sur y este del Mediterráneo se comprometían a la progresiva liberalización de sus sistemas comerciales y a la reforma de sus economías "thereby the EU was actively promoting the Washington consensus". 93 Además de la promoción de una economía de libre mercado, la provisión de la ayuda comunitaria aparecía ligada o condicionada por la agenda normativa de la UE. El conjunto de valores democráticos y liberales sobre los que se cimienta la UE (la democracia, los derechos humanos, el Estado de derecho y el buen gobierno) quedan reflejados como requerimientos indispensables (o condiciones) en los documentos relativos a la AEM, tanto en la Declaración final de Barcelona como en cada uno de los Acuerdos de Asociación, sustitutos de los acuerdos de cooperación vistos más arriba. ${ }^{94}$

En este sentido algunos autores consideran que el proceso de Barcelona "served as the foundations for a long-term exercise in soft power projection". Si el ambicioso objetivo oficial era convertir la cuenca del Mediterráneo en un área de intercambio, paz y estabilidad, el objetivo no declarado "was to defuse migratory pressures from the South by creating stability and supporting economic development". ${ }^{95}$ Es decir, que los objetivos implícitos de la UE serían:

"To forestall or contain potential security problems arising from challenges such as violent conflict within these states; nationalist and fundamentalist political ideologies; socio-economic discontent; and mass migration. (...) Politically, the EU has sought to promote an agenda of good governance, democratization and respect for human rights". ${ }^{96}$

El término mismo de 'asociación' o 'partenariado' ha sido criticado por algunos autores, dada la asimetría y desigualdad que caracteriza las relaciones entre ambas orillas del Mediterráneo y el papel primordial que desempeña la Unión Europea tanto en la formulación de las prioridades políticas como en el establecimiento de condiciones a los países de la ribera sur. Las motivaciones de carácter securitario han guiado la acción de la UE en la región, cuya preocupación por la inestabilidad política y la posibilidad de fenómenos migratorios que afectaran a la orilla norte fomentó el desarrollo del nuevo marco institucional de las relaciones euro-mediterráneas y la aportación de generosos recursos económicos para atajar dicha situación. ${ }^{97}$

\footnotetext{
${ }^{92}$ Holden, Patrick: "Security, power or profit? The economic diplomacy of the US and the EU in North Africa", The Journal of North African Studies, vol. 14, nº1 (Marzo 2009), pp. 21-22.

${ }^{93}$ Whitman, op.cit.

${ }^{94}$ Tanner, op. cit., p. 137.

95 Ibid., p.137.

${ }^{96}$ Cottey, Andrew (2007): Security in the new Europe, The new Europe series, Basingstoke, Palgrave Macmillan, p. 88.

97 Holden, Patrick: "Partnership Lost? The EU's Mediterranean Aid Programmes", Mediterranean Politics, vol.10, no 1 (Marzo 2005), pp. 19-22.
} 


\subsection{Las causas del fracaso del proceso}

A pesar del enfoque ambicioso del proceso de Barcelona, de su naturaleza holística y de la formulación y diseño de nuevos mecanismos e instrumentos, el balance de la AEM - y su sucesor institucional en 2008, la Unión por el Mediterráneo - no es muy positivo. No se consiguió dar el impulso esperado a las relaciones euro-mediterráneas ni se avanzó en la integración regional de las riberas sur y este del Mediterráneo.

Un factor fundamental que socavó el proceso fue la reanudación de las hostilidades en el conflicto árabe-israelí lo cual impidió la cooperación en materia política y de seguridad:

“(...) Israel is a signatory of the Barcelona Declaration, and it was hoped that its participation in the Euromed process would pave the way towards a final settlement of its conflict with its Arab neighbours. However, its behaviour since then has been the major reason for bringing the Barcelona Process to a complete halt". ${ }^{98}$

Además de las limitaciones de la UE en materia de resolución de conflictos, el impulso económico en la región avanzó de forma lenta y no se vio correspondido por el desarrollo social y democrático. De hecho las condiciones internas de los países de la ribera sur se vieron deterioradas, contradiciendo las expectativas europeas que aunaban las condiciones socioeconómicas con procesos de democratización. ${ }^{99}$ La condicionalidad política, elemento fundamental de la asociación euro-mediterránea, que preveía la posible suspensión de la ayuda financiera de la Comunidad en el caso de violaciones de los derechos humanos, no supuso tampoco un incentivo para los gobiernos de los países socios ni funcionó como elemento de presión por parte de la UE.

"The refusal of many of these governments to respect their engagements was paralleled by the reluctance of the EU to use the Barcelona provisions to exert pressure on the states that failed to observe these principles". ${ }^{100}$

En el ámbito de la integración regional, una de las prioridades del partenariado, los avances fueron prácticamente nulos. Los socios mediterráneos no constituyen una única región homogénea e integrada por lo que la vertiente multilateral del proceso de Barcelona quedó desdibujada favoreciendo el énfasis en la firma de acuerdos bilaterales con cada tercer país mediterráneo, dificultando así el desarrollo de una verdadera zona de libre comercio:

"All of the partnership agreements in existence involve two signatories: the EU acting as a single entity on behalf of its member states, on the one hand, and an individual MPC acting on its own behalf, on the other (...) which gives us a set of 11 agreements mutually binding for the $E U$ and each one of its partners but not applicable to horizontal relations between MPCs". ${ }^{101}$

\footnotetext{
98 Chourou Béchir: "Arab Regional Integration as a Prerequisite for a Successful Euro-Mediterranean Partnership", Mediterranean Politics, vol. 8, n 2-3 (2003), p. 212.

99 Tanner, op.cit., pp. 139-140; Dannreuther, Roland (2008): “The European Security Strategy's regional objective. The neighbourhood policy", en Biscop, Sven y Andersson, Jan Joel (eds.) The EU and the European Security Strategy - Forging a global Europe, London, Routledge, p. 68.

${ }^{100}$ Keukeleire y MacNaughtan, op. cit., p. 276.

${ }^{101}$ Chourou, op. cit., p. 195.
} 
En consecuencia, los intercambios comerciales en la cuenca mediterránea no se realizan a nivel horizontal entre los países de la ribera sur, sino que se manifiestan de forma bilateral entre la UE y cada uno de los socios o terceros países mediterráneos. Algunas de las razones que explican la limitación de intercambios sur-sur serían

"The lack of complementarity in their production, the existence of very high tariff protection, heavy administrative regulations and, in some cases (...) the fact that their markets are small and fragmented and their economies not diversified". ${ }^{102}$

\subsection{La decepción en el sector agrícola}

En el ámbito de la agricultura y la seguridad alimentaria, y de forma general, el problema de fondo del Proceso de Barcelona, como sostienen algunos autores:

"Is the implementation gap between rhetoric and policy and the contradictions with the liberal philosophy behind the projection of soft security. The EU, internally constrained by the Common Agricultural Policy (CAP) and farmer lobbies from South European states, restricts the import of agricultural products from the South and applies free trade only to oil, gas and industrial products. Even in the textile sector, the South has been pressurized to accept 'voluntary restraint agreements', while exposing previously protected domestic sectors 'to the full force of European competition',". ${ }^{103}$

El saldo comercial entre las dos orillas del Mediterráneo ha sido siempre favorable para la UE, excepto en el caso de los países de los que la UE importa recursos energéticos. Ello se debe a la naturaleza de las exportaciones (productos manufacturados) de la UE a dichos países. Otro obstáculo para el desarrollo económico de los países del norte de África sería la "excessive dependence on the export of one or two commodities - oil and gas in Algeria, phosphates in Morocco, textiles in Tunisia". De ahí el anhelo de dichos países de desarrollar su potencial agrícola mediante el acceso al mercado europeo. ${ }^{104}$

Podemos concluir esta sección afirmando que con la firma de los acuerdos de asociación con los socios mediterráneos ${ }^{105}$ la UE intentaba promover, por un lado, una política comercial neoliberal, eliminando las barreras y restricciones comerciales que existían en relación a los intercambios de productos manufacturados; por otro lado, la UE enviaba un mensaje contradictorio a los terceros países mediterráneos excluyendo de los acuerdos el comercio de productos agrícolas, en el que los socios mediterráneos presentaban ventajas comparativas. 106

\footnotetext{
102 Tovias, Alfred y Bacaria, Jordi: “Free trade and the Mediterranean”, Mediterranean Politics, vol. 4 , $\mathrm{n}^{\mathbf{0}} 2$ (Verano 1999), p. 6.

${ }^{103}$ Joffé, G., citado en Tanner, op. cit., p. 140.

${ }^{104}$ Tovias y Bacaria, op. cit., pp. 8, 13, 14.

105 Los acuerdos euromediterráneos de asociación con los países del norte de África que nos interesan se firmaron y entraron en vigor en las fechas siguientes:

Argelia: Firmado el 22/04/02; entrada en vigor el 01/09/05

- Marruecos: Firmado el 26/02/96; entrada en vigor el 01/03/00

- $\quad$ Túnez: Firmado el 17/07/95; entrada en vigor el 01/03/98

Fuente: http://ec.europa.eu/trade/creating-opportunities/bilateral-relations/regions/euromed/index_en.htm

${ }^{106}$ Keukeleire y MacNaughtan, op.cit., p.277; Tovias y Bacaria, op. cit., pp.3-4.
} 
Así pues, el "talón de Aquiles" del partenariado era precisamente el hecho de no incluir los productos agrícolas:

"The consequences are of two kinds: the exclusion can diminish the potential gains of the agreement, and can slow the economic development in the Mediterranean countries because they are encouraged to reinforce tradition low-value added productivity sectors". ${ }^{107}$

No obstante, la evolución del proceso de Barcelona ha traído consigo una progresiva apertura del mercado europeo en lo que respecta al sector agrícola, en grados variables en función de la evolución de las relaciones bilaterales y de los acuerdos firmados con cada uno de los socios mediterráneos:

\begin{abstract}
"Improving their access to the European agricultural markets is a major concern for SEMCs [Southern and Eastern Mediterranean Countries]. Since the 1995 Barcelona Declaration, trade liberalization has been the cornerstone of the EU Euro-Mediterranean policy, with an ambitious initial objective to create a free trade area between the EU and most SEMCs. In 2005, it was decided to relaunch the process through gradual but full liberalization of trade in agricultural products, except for a limited number of sensitive products". ${ }^{108}$
\end{abstract}

\title{
4. La Política Europea de Vecindad: objetivos, principios, aportaciones y limitaciones; imarco político novedoso o "vino viejo en odres nuevos"?
}

El fracaso del Proceso de Barcelona y de sus tres asociaciones o partenariados llevó a la UE a un proceso de reflexión en torno a nuevas modalidades y estrategias para superar el intento fallido de establecer un marco de relaciones comprehensivo entre las dos orillas del Mediterráneo.

Aunque en un principio los 'vecinos del sur' no eran el objeto prioritario de la discusión, debido a la ampliación de 2004 y la oscilación del centro de gravedad de las preocupaciones europeas hacia el este de Europa, el foco de interés se extendió a los países de la cuenca mediterránea para, por un lado, contrarrestar y equilibrar la predominancia de las propuestas políticas para los 'vecinos del este'; y por otro lado, para reformar y dar un nuevo ímpetu a las relaciones euro-mediterráneas.

Sin embargo, la Política Europea de Vecindad (PEV) no nace como una política totalmente diferente sino como un continuum; ni sustituye al Proceso de Barcelona, sino que lo complementa y fortalece, manteniendo los principales principios, estrategias y objetivos de las anteriores aproximaciones y políticas de la UE hacia el Mediterráneo. Si bien el instrumento financiero MEDA de la AEM es sustituido en 2007 por el nuevo Instrumento Europeo de Vecindad y Asociación (IEVA o ENPI), la PEV no sustituye o revisa en profundidad las anteriores medidas e instrumentos, sino que mantiene (y amplía) las políticas

\footnotetext{
${ }^{107}$ Guerrieri, Paolo y Caratelli, Irene: "EU's Regional Trade Strategy, the Challenges Ahead", The International Trade Journal, vol. 20, nº 2 (Summer 2006), p. 149.

${ }^{108}$ Emlinger, Charlotte; Chevassus-Lozza, Emmanuelle y Jacquet, Florence: "Fruit and vegetable access to EU markets: Dissecting tariffs faced by Mediterranean countries”, Food Policy, no 35 (2010), p. 599.
} 
e instituciones creadas anteriormente y los Acuerdos euro-mediterráneos de asociación, no obstante complementados por los nuevos Planes de Acción de la PEV. ${ }^{109}$

"The inclusion of new areas of cooperation in the ENP mainly mirrored the EU's expanding policy competences and widening policy agenda since the 1995 Barcelona Conference and since the original Association Agreements were agreed, rather than symbolizing a considered policy development. The EU's strategy was paradoxically becoming more inward looking". ${ }^{110}$

\subsection{Un largo proceso de discusión: las bases de la PEV}

El proceso de reflexión y formulación de la PEV se desarrolló entre 2002 y 2004, fecha de publicación del documento estratégico de la nueva política.

En mayo de 2002, el entonces ministro de Asuntos Exteriores británico, Jack Straw, envió una carta a la presidencia española del Consejo Europeo. Consciente de los retos que supondría la ampliación hacia el este de la Unión Europea, tanto en lo que respecta a la accesión de los nuevos países miembros como en lo referente a la cercanía de nuevos vecinos, Straw proponía nuevas políticas para tres de las repúblicas ex-soviéticas, de tal forma que se evitaran situaciones de inseguridad que pudieran afectar a la futura Europa ampliada:

"We also need to look further east to the EU's new neighbours. We must not create a new dividing line of 'haves and have not's' on the continent. I have recently written to my EU counterparts making the case for new policies towards Ukraine, Moldova and Belarus which offer incentives for reform". 111

Tras la carta de Straw se inició un proceso de reflexión y de formulación de diferentes propuestas e iniciativas que desembocarían en la institucionalización de la política europea de vecindad de forma oficial en 2004.

En agosto de 2002, el Alto Representante de la UE para la PESC, Javier Solana, y el entonces Comisario de Relaciones Exteriores, Christopher Patten, formularon la iniciativa "Wider Europe" (una Europea ampliada) en una carta conjunta que exploraba la nueva situación creada por la perspectiva de la ampliación a la hora de desarrollar relaciones con los nuevos vecinos de la UE. El texto supuso la ampliación del debate, en origen centrado en los países del este de Europa, al incluir en la iniciativa a los países de los Balcanes occidentales y de la ribera sur del Mediterráneo ${ }^{112}$ :

"The starting point should be that relations with all our neighbours should be based on a shared set of political and economic values. Building on this, we should aim towards regional stability and co-operation, closer trade links and approximation and/or harmonisation of legalisation and progressive extension of all relevant EU policies. Looking to the medium and

\footnotetext{
${ }^{109}$ Keukeleire y MacNaughtan, op. cit., p. 279; Dannreuther, op. cit., p. 68.

${ }^{110}$ Keukeleire y MacNaughtan, op. cit., p. 279.

${ }^{111}$ Straw, Jack (2002): Speech “A New Mission for Europe”, 27 Mayo 2002, Berlin, en http://collections.europarchive.org/tna/20080205132101/http://www.fco.gov.uk/servlet/Front?pagename=Open Market/Xcelerate/ShowPage\&c=Page\&cid=1063632562982\&a=KArticle\&aid=1022256741983.

${ }^{112}$ La mención de los Balcanes refleja el estado del debate sobre el nuevo marco político, no separando claramente la política de ampliación de la política de vecindad. En documentos posteriores se aclara la distinción y se delimita la zona de interés de la PEV.
} 
longer term, we could foresee a gradually evolving framework for an economic and political space surrounding the Union, which would nevertheless stop short or full membership or creating shared institutions. Building on existing instruments and relations, this approach could ultimately bring neighbouring countries fully into the internal market and other relevant EU policies". ${ }^{113}$

A finales del mismo año, en diciembre de 2002, el Presidente de la Comisión, Romano Prodi, profundizó en la iniciativa "Wider Europe", definiendo una 'política de proximidad' como la clave para la estabilidad de la Europa ampliada:

"My aim is giving them incentives, injecting a new dynamic in existing processes and developing an open and evolving partnership. This is what we call our proximity policy, a policy based on mutual benefits and obligations, which is a substantial contribution by the EU to global governance". ${ }^{114}$

En su discurso, Prodi menciona algunas de las bases fundamentales de la PEV y también algunos de los términos más frecuentemente utilizados en la retórica de la política de vecindad, como la referencia a la proximidad geográfica (y a los efectos perniciosos que puede acarrear) y al "círculo de amigos" alrededor de las nuevas fronteras de Europa:

"The geographical scope of this approach is our neighbourhood in the literal sense of the word, our backyard. It includes our future eastern neighbours and the whole Mediterranean area (...) I want to see a 'ring of friends' surrounding the Union and its closest European neighbours, from Morocco to Russia and the Black Sea". 115

Así como la naturaleza de las 'relaciones privilegiadas' con los países vecinos, expresada en la ambiciosa frase "compartir todo excepto las instituciones", excluyendo de esta forma la posibilidad de membresía y diferenciando la política de vecindad de la política de ampliación, aunque ofreciendo relaciones de un alcance mayor que una asociación:

"sharing everything with the Union but institutions. The aim is to extend to this neighbouring region a set of principles, values and standards which define the very essence of the European Union". 116

La síntesis comprehensiva de las discusiones y debates en torno a la elaboración de la nueva política, así como la definición del marco institucional de las relaciones de la UE con sus vecinos ante la perspectiva de la ampliación en 2004, vieron la luz en dos documentos elaborados por la Comisión en marzo de 2003 (Una Europa más amplia. Relaciones con los

113 Patten, Christopher y Solana, Javier (2002): Carta conjunta "Wider Europe", en http://www.lfpr.lt/uploads/File/2002-10/Letter.pdf.

${ }^{114}$ Prodi, Romano (2002): Speech “A Wider Europe - A Proximity Policy as the key to stability", Sixth ECSAWorld Conference, Jean Monnet Project; 6 Diciembre 2002, Brussels, en http://europa.eu/rapid/pressrelease SPEECH-02-619 en.htm.

115 Ibid.

${ }^{116}$ Ibid. 
países vecinos: un nuevo marco para las relaciones con nuestros vecinos del Este y del Sur de Europa) y mayo de 2004 (Política europea de vecindad: Documento de Estrategia). La prioridad estratégica de las relaciones de la UE con sus vecinos se mencionó también en la Estrategia Europea de Seguridad de 2003, donde se identificaban las principales amenazas a la seguridad europea, así como la necesidad de promover la estabilidad en los países vecinos.

\title{
4.2. Objetivos, principios y novedades fundamentales
}

El documento estratégico de la 'Política Europea de Vecindad' establece que el objetivo de la política es:

\begin{abstract}
“compartir los beneficios de la ampliación de la UE en 2004 con los países vecinos, mediante el refuerzo de la estabilidad, de la seguridad y del bienestar para todas las partes afectadas. Está pensada para prevenir la aparición de nuevas líneas divisorias entre la UE ampliada y sus vecinos y ofrecer a éstos la oportunidad de participar en diversas actividades de la UE gracias a una mayor cooperación en los ámbitos de la política, la seguridad, la economía y la cultura". 117
\end{abstract}

Los documentos de 2003 y 2004 definen la naturaleza de la 'política de vecindad', formulando el enfoque y la visión del proceso, incorporando los principales elementos de las discusiones previas (la promoción de la estabilidad, la diferencia de la política de vecindad con la política de ampliación al excluir la posibilidad de accesión a la UE, el 'círculo de amigos' en torno a la Unión y la participación en el mercado interno) así como estableciendo de forma estructurada los objetivos, los mecanismos de negociación y supervisión, los incentivos financieros y los pasos a seguir.

Una de las innovaciones respecto a los marcos de relaciones euro-mediterráneas anteriores es la insistencia en la elaboración de un enfoque gradual y en que el principio de diferenciación entre los países constituirá "la base de la nueva política de vecindad". Dado que "los países vecinos no parten de un mismo punto en sus relaciones con la UE", se abre la puerta al desarrollo de relaciones con cada uno de los vecinos a un ritmo diferente en función del grado de implicación y de los avances realizados. Los principios de diferenciación y progresividad se establecerán mediante planes de acción específicos para cada país, propuestos por la Comisión y acordados con el país respectivo, "a fin de exponer claramente los objetivos estratégicos globales y los criterios de referencia con los que se puedan evaluar los avances en un período de varios años". ${ }^{118}$

Los planes de acción definen la agenda y los pasos a seguir, así como los ámbitos prioritarios de actuación, incluyendo "diálogo y reforma política; intercambios comerciales y medidas que preparen a los socios para aumentar gradualmente su participación en el mercado interior de la UE; justicia y asuntos de interior; energía, transporte, sociedad de la información, medio ambiente, investigación e innovación; así como política social y contactos entre comunidades ("persona a persona")". ${ }^{119}$ No obstante la oferta de "relaciones privilegiadas', la PEV no define claramente en función de qué criterios son definidas las

\footnotetext{
${ }^{117}$ Comisión de las Comunidades Europeas (2004a): "Política europea de vecindad. Documento de Estrategia", Comunicación de la Comisión, 12 Mayo 2004, COM (2004) 373 final, p.3.

${ }^{118}$ Comisión de las Comunidades Europeas (2003): "Una Europa más amplia. Relaciones con los países vecinos: un nuevo marco para las relaciones con nuestros vecinos del Este y del Sur de Europa”, Comunicación de la Comisión al Consejo y al Parlamento Europeo, 11 marzo 2003, COM (2003) 104 final, p.17.

${ }^{119}$ Comisión (2004a), op.cit., p.3
} 
prioridades ni cuáles son los incentivos o ventajas adquiridos a medida que se van cumpliendo los objetivos de reforma debido a la falta de precisión sobre "the exact benefits gained by their fulfillment". 120

A pesar de que la lógica de actuación es similar a la de la política de ampliación en el sentido de que "the deepening of the relationship through the Action Plans is made conditional on their implementation" ${ }^{121}$, la condicionalidad por sí sola, sin la prospectiva de la ampliación, no puede conseguir la proyección del modelo europeo ni puede explicar "the success or failure of rule transfer from the EU to the ENP countries". ${ }^{122}$

Un segundo aspecto fundamental y llamativo de la PEV es la propuesta a los países vecinos de participar en las cuatro libertades fundamentales de la UE de circulación de personas, mercancías, servicios y capital. Ante la ausencia del incentivo que supone la posible accesión como países miembros, la UE propone como oferta atractiva el acceso al mercado interno y las cuatro libertades de la Unión:

"Más concretamente, debería ofrecerse a todos los países vecinos la perspectiva de participar en el mercado interior de la UE y de continuar con la integración y la liberalización para impulsar la libre circulación de personas, mercancías, servicios y capitales (las cuatro libertades)". ${ }^{123}$

Sin embargo, no encontraremos esta referencia en documentos posteriores, ni su realización práctica, sino la continuidad de la protección del mercado interno en lo relativo a productos sensibles para la Unión, y la persistencia del control de los flujos migratorios provenientes de los países vecinos. Dannreuther considera que la formulación del incentivo de las cuatro libertades es de naturaleza 'evolutiva', favoreciendo una mayor apertura de forma gradual y que:

"The main innovation and progress is more on the procedural than the substantive level in that the ENP offers greater support, particularly of a technical nature, in ensuring that neighbouring countries come closer to approximating international and EU regulatory standards so that they are less vulnerable to EU non-tariff barriers, such as anti-dumping measures". ${ }^{124}$

En tercer lugar, la PEV pretende ser una política "coherente y eficiente", superando la complejidad y división reinantes a la hora de implementar las políticas regionales de los años anteriores, lo cual provocó la confusión y la ineficiencia de los programas e instrumentos en las regiones de interés. La nueva política integra "los componentes afines de los tres 'pilares' de la actual estructura de la Unión” lo cual "permitirá a los países vecinos compartir los

\footnotetext{
${ }^{120}$ Dannreuther, op. cit., p. 70.

121 Edwards, Geoffrey(2009): "The Construction of Ambiguity and the Limits of Attraction: Europe and its Neighbourhood Policy", en Jones, Erik y van Genugten, Saskia (eds.): The Future of European Foreign Policy, Oxon, Routledge, p. 45.

122 Casier, Tom (2010): "The European Neighbourhood Policy: Assessing the EU's Policy toward the Region", en Bindi, Federiga (ed.): The Foreign Policy of the European Union: Assessing Europe's Role in the World, Washington DC, Brookings Institution Press, p. 102.

${ }^{123}$ Comisión (2003), op. cit. p. 10.

${ }^{124}$ Dannreuther, op. cit., p. 69.
} 
beneficios de la ampliación de la UE en lo tocante a la estabilidad, la seguridad y el bienestar". 125

En lo que respecta a los países de la cuenca mediterránea, la PEV no sustituye el marco de la asociación euro-mediterránea, sino que la complementa, colaborando en la consecución de los objetivos de la asociación estratégica para el Mediterráneo. De hecho "la política europea de vecindad propiamente dicha se aplicará a través del proceso de Barcelona y los Acuerdos de Asociación con cada país socio". ${ }^{26}$ Para ello, se dotó a la nueva política de mayores recursos, ascendiendo el presupuesto del Instrumento Europeo de Vecindad y Asociación (IEVA) a 11.181 millones de euros para el período 2007-2013, lo cual significa un aumento considerable respecto a los fondos asignados a los programas TACIS y MEDA (3.138 y 5.350 millones de euros respectivamente para el periodo 2000-2006). ${ }^{127}$

\subsection{Las contradicciones e intereses subyacentes}

Uno de los objetivos formales de la PEV es el de "reducir la pobreza y crear una zona de prosperidad y valores compartidos"128 para "prevenir la aparición de nuevas líneas divisorias entre la UE ampliada y sus vecinos". ${ }^{129}$

En este sentido, podemos argumentar que los cambios en el escenario internacional de la post-Guerra fría han condicionado la visión de la UE a la hora de formular su política de vecindad, en la que se manifiesta una concepción de la seguridad vinculada al desarrollo que ha traído consigo un cambio gradual en las relaciones con los países de la orilla sur del Mediterráneo, primando los intereses de estabilidad europeos (la protección frente a las 'amenazas provenientes de dichos países') sobre los objetivos de desarrollo socio-económico en la región:

"The increasing prominence of the EU's neighbourhood policies reflects the changed nature of security within Europe. In contrast to the Cold War, many of the primary security challenges facing Europe at the beginning of the twenty-first century arise not from direct military threats to European states but rather from the potential consequences of political and socio-economic instability on Europe's periphery (...) the EU faces various combinations of resistance to reform, unresolved or ongoing conflict, authoritarian or semi-authoritarian regimes, and a wariness about external interference. In these circumstances, the EU's ability to shape developments in these regions is often limited". ${ }^{30}$

Sin embargo, la ausencia del 'premio' del ingreso en la UE ha obstaculizado el margen de maniobra de la Unión a la hora de intentar imponer reformas y de exportar el modelo y las normas europeas a terceros países, en un intento de 'promotion of Europeanisation by

\footnotetext{
${ }^{125}$ Comisión (2004a), op.cit., p.6.

${ }^{126}$ Ibid., pp. 6-7.

${ }^{127}$ Fuentes: Para el IEVA

http://europa.eu/legislation_summaries/external_relations/relations_with third_countries/eastern_europe and ce ntral_asia/r17101_es.htm; para MEDA:

http://europa.eu/legislation_summaries/external_relations/relations_with third_countries/mediterranean_partner countries/r15006_en.htm; para TACIS:

http://europa.eu/legislation_summaries/external_relations/relations_with third_countries/eastern_europe and_ce ntral_asia/r17003 en.htm.

${ }^{128}$ Comisión (2003), op. cit., p. 9.

${ }^{129}$ Comisión (2004a), op. cit., p. 3.

${ }^{130}$ Cottey, op. cit., p. 91.
} 
recognising that trade, aid and development, key areas of its business, are the core of the EU's claim to international actorness". 131

La agenda normativa de la exportación de las normas y valores de la UE presenta ventajas indiscutibles para la Unión, pero los intereses estratégicos que motivan la acción europea han sido criticados por recaer en una aproximación predominantemente securitaria de las relaciones con los países vecinos, centrada en las 'amenazas', en lugar de favorecer una agenda estructural y transformadora. ${ }^{132}$

En su carácter englobante de varios pilares, la PEV manifiesta su relación indisoluble con los asuntos de seguridad y su relación con la Estrategia Europea de Seguridad, documento discutido y formulado simultáneamente al debate sobre 'una Europea más amplia', que incide particularmente en las amenazas a la seguridad de Europa que pueden surgir de la proximidad con los países vecinos: ${ }^{133}$

"A Europa le conviene que los países limítrofes estén bien gobernados. Los vecinos inmersos en conflictos violentos, los Estados débiles en los que prolifera la delincuencia organizada, las sociedades disfuncionales o las explosiones demográficas en nuestras fronteras plantean problemas a Europa". ${ }^{134}$

Por otro lado, la aspiración de crear un 'anillo de amigos' en torno al espacio geográfico de la UE, en palabras de la Estrategia Europea de Seguridad (EES), la promoción de "un conjunto de países bien gobernados al este de la Unión Europea y en las orillas del Mediterráneo"135 es de difícil consecución al no existir el incentivo de la posibilidad de la accesión a la Unión. La aceptación de las normas y valores de la Unión a cambio del acceso al mercado interno es la forma de la UE de exportar su propio modelo de estabilidad sin abrirlo a los nuevos socios, generando tensiones en la aceptación de tales normas y alejando el proceso de la posibilidad de constituir una asociación compartida, exacerbando la asimetría de las relaciones. ${ }^{136}$

Además, la supeditación de la PEV a intereses securitarios ha provocado que la Unión enviara mensajes contradictorios a los vecinos del norte de África, cuyas demandas de un mayor acceso al mercado único europeo en el sector agrícola y la libertad de movimiento de las personas han estado tradicionalmente ausentes de la agenda y de los planes de acción, aunque el 'principio de diferenciación' promovido por la PEV ha favorecido diferentes velocidades y ha tenido diferentes consecuencias en cada uno de los países vecinos.

\footnotetext{
${ }^{131}$ Jones, Alun y Clark, Julian: "Europeanisation and Discourse Building: The European Commission, European Narratives and European Neighbourhood Policy", Geopolitics, vol. 13, n³ (2008), p. 547.

${ }^{132}$ Dannreuther, op. cit., pp. 72-73.

${ }^{133}$ Ver también al respecto Dannreuther, op. cit., p. 70 y Edwards (2009), op. cit., p. 45.

${ }^{134}$ Consejo Europeo (2003): Una Europa segura en un mundo mejor. Estrategia Europea de Seguridad, $12 \mathrm{de}$ diciembre de 2003, p.7, en http://www.consilium.europa.eu/uedocs/cmsUpload/031208ESSIIES.pdf.

${ }^{135}$ Ibid., p. 8.

${ }^{136}$ Casier, op. cit., p. 105 y Edwards (2009), op. cit., pp. 48-49.
} 


\subsection{El enfoque diferenciado y su impacto variable en los casos marroquí, argelino y tunecino}

\subsubsection{Marruecos}

Si hay un caso en el que el impacto de la PEV haya sido llamativo es el de Marruecos, el 'buen alumno' que ha visto recompensados sus esfuerzos con incentivos y con el fortalecimiento de sus relaciones bilaterales con la UE tanto cuantitativa como cualitativamente.

El Acuerdo de Asociación, firmado en el marco de la asociación euro-mediterránea en 1996 (en vigor el 1 de marzo de 2000), constituye el marco jurídico de las relaciones entre la Unión Europea y Marruecos, y sentó las bases para el establecimiento progresivo de una zona de libre comercio para productos manufacturados, comenzando el proceso en 2003 y dando por concluido el periodo de supresión de aranceles en marzo de 2012. ${ }^{137}$ En este marco, Marruecos ha sido el principal beneficiario de la asistencia comunitaria bajo el programa MEDA, recibiendo un total de 1.181.3 millones de euros durante el periodo 1995-2003. ${ }^{138}$

Este marco se vio complementado en 2005 con la aprobación del Plan de Acción de la PEV, destinado a profundizar las relaciones bilaterales tanto en el ámbito económico, como en el político, social y cultural, con el fin de acometer reformas estructurales en el terreno económico y político, y con la perspectiva en el ámbito agrícola de converger "towards the necessary conditions for establishment of a Morocco-EU free trade area", aunque las negociaciones sobre agricultura han sido siempre difíciles y han entorpecido en gran medida las relaciones de la UE con Marruecos. ${ }^{139}$

Antes del plazo del vencimiento del Plan de Acción en 2010 comenzaron las negociaciones para evaluar la posibilidad de otorgar a Marruecos un 'estatuto avanzado', en función de la progresión de las reformas acometidas, cuya concesión se oficializó en octubre de 2008. En septiembre de 2011 la UE anunció la apertura de negociaciones para lograr gradualmente el establecimiento de una zona de libre comercio "profunda y comprehensiva" con Marruecos. ${ }^{140}$

Y en febrero de 2012 se firmó, hito de las relaciones de la UE con Marruecos en el marco de la política europea de vecindad, el "Acuerdo UE-Marruecos sobre el comercio de productos agrícolas, productos agrícolas transformados y la pesca”, que entró en vigor en octubre del mismo año. ${ }^{141}$

El acuerdo "will immediately reduce or remove 55\% of tariffs on Morocco agricultural and fisheries products (up from 33\%) and 70\% of tariffs on EU agricultural and fisheries products within 10 years (rising from 1\%)" e incluye algunas salvaguardas en lo que respecta a los productos más sensibles "by allowing only moderate increases to quotas of certain products, such as tomatoes, strawberries, cucumbers and garlic. The deal also provides for

\footnotetext{
137 EU-Morocco Euro-Mediterranean Agreement (2000), Official Journal of the European Communities, 18 Marzo 2000, L70, pp. 2-190. Y "La zone de libre échange UE-Maroc pour les produits industriels devient effective (29/02/2012)", Press Release, Délégation de l’UE au Royaume du Maroc, 1 Marzo 2012, en http://eeas.europa.eu/delegations/morocco/press_corner/all_news/news/2012/20120229fr.htm\#.

${ }_{138}$ Commission of the European Communities (2004b): "European Neighbourhood Policy. Country Report Morocco" \{COM (2004)373 final\}, Commission Staff Working Paper, 12 Mayo 2004, SEC (2004) 569, p.4.

${ }^{139}$ EU-Morocco Action Plan, op. cit. p. 9.

${ }^{140}$ Deep and Comprehensive Free Trade Agreement (DCFTA).

${ }^{141}$ Véase http://ec.europa.eu/trade/creating-opportunities/bilateral-relations/countries/morocco/.
} 
seasonal quotas to counter distortion of the EU market and says Moroccan imports should meet European sanitary standards". ${ }^{142}$

Aunque el acuerdo podría garantizar el desarrollo del potencial agrícola de Marruecos y un mayor desarrollo económico, algunas críticas han surgido en relación a la explotación de los territorios disputados del Sáhara Occidental, desproveyendo a los saharauis de sus recursos naturales, lo que fomentaría una situación de inseguridad alimentaria; y en las dudas sobre la posición que jugarán los pequeños productores, para los que este acuerdo puede representar una desventaja frente a la competencia de compañías transnacionales. ${ }^{143}$

El principio de diferenciación promulgado por la política europea de vecindad fue muy bien acogido por Marruecos, que se mostró 'muy cooperativo' en la implementación de la política europea de vecindad realizando importantes progresos para garantizar la intensificación de las relaciones privilegiadas con la UE, su principal socio comercial:

"Morocco particularly welcomes the prospect of a bilateral, differentiated approach that takes account of the degree of political will and actual capacity exhibited by each partner, with a view to developing relations in a way which accurately reflects each country's specific situation. In this respect, the strategic use of the neighbourhood policy is precisely what Morocco has asked for". ${ }^{144}$

Además, Marruecos se ha mostrado muy activo en el Consejo de la Asociación EuroMediterránea y en otros marcos institucionales como el grupo $5+5$, la Unión del Magreb Árabe y el Acuerdo de Agadir de 2004. Estos intentos de cooperación sur-sur y de acercarse a la UE, junto con los otros elementos mencionados, han tenido sus frutos. ${ }^{145}$

Particularmente relevante es la cooperación de Marruecos en materia de seguridad. En este terreno, la participación de las fuerzas armadas reales marroquíes en la Operación ALTHEA en Bosnia, bajo mando de una fuerza multinacional de la Unión Europea (EUFOR) desde finales de 2004 simboliza la voluntad de Marruecos "de desarrollar de manera multilateral una cooperación militar eficiente tanto con la OTAN como con la UE"146 y la cooperación con las estructuras de seguridad europeas, "as the operation was under the supervisión of the Political and Security Committee in the Council of EU hierarchy, Morocco was de facto participating in an ESDP mission". ${ }^{147}$

142 European Parliament News: "Green light for EU-Morocco trade deal", 16 Febrero 2012, en http://www.europarl.europa.eu/news/en/pressroom/content/20120216IPR38354/html/Green-light-for-EUMorocco-trade-deal.

${ }^{143}$ Véanse: "Los servicios legales del PE cuestionan pacto agrícola con Marruecos, WSRW, 2 Febrero 2011, en http://wsrw.org/a110x1845 y EU-Mediterranean, bilaterals.org, en http://www.bilaterals.org/spip.php?rubrique173\&lang=en.

${ }_{144}$ Commission of the European Communities (2004b), op . cit., p. 5; "European Neighbourhood Policy. Country Report Morocco" \{COM (2004)373 final\}, Commission Staff Working Paper, 12 Mayo 2004, SEC (2004) 569. p.5.

${ }_{145}$ Bicchi, op. cit., pp. 209-210.

${ }^{146}$ Marsou, Mokhtar: "La participación de las fuerzas armadas reales marroquíes en la operación ALTHEA“, $C I D O B$, V Seminario Internacional sobre Seguridad y Defensa en el Mediterráneo, p. 55.

${ }^{147}$ Bicchi, op. cit., p. 212. 
Podemos concluir afirmando que el caso marroquí representa un 'caso ejemplar' a los ojos de la UE en su implementación de la PEV, a pesar de las restricciones a la entrada en el mercado interno de los productos agrícolas procedentes de Marruecos y de la persistencia de capítulos conflictivos como el del control migratorio, así como la dependencia energética de la Unión en cuanto a la situación de Marruecos como país de tránsito en el Magreb.

Aunque en términos generales la evaluación es positiva, el patrón de primacía de los intereses securitarios de la UE se refleja en la preocupación predominante sobre el control de fronteras y la inmigración ilegal, a la cual están supeditados de una manera u otra los demás objetivos:

"What is however striking in EU-Morocco relations is the low priority of political issues in comparison with economic (and energy related) issues. An important exception to this rule is the area of Justice and Home Affairs, where arguably, the politics of insecurity produces policies at incomparable speed (...) illegal immigration is pictured as the principal concern of the EU member states and Morocco". ${ }^{148}$

\subsubsection{Túnez}

Túnez fue el primer país mediterráneo en firmar un Acuerdo de Asociación en el marco de la AEM, en julio de 1995 (en vigor en marzo de 1998) y fue también el primer socio mediterráneo que finalizó el proceso de supresión de aranceles para productos manufacturados, estableciendo una zona de libre comercio en 2008. ${ }^{149}$ Para la consecución de este objetivo, Túnez recibió un montante de 734 millones de euros procedente de los fondos MEDA para el periodo 1995-2003. ${ }^{150}$

Al igual que Marruecos, Túnez firmó el Acuerdo de Agadir con Egipto, Marruecos y Jordania en febrero de 2004 (entrando en vigor en julio de 2006), con el objetivo de favorecer los intercambios comerciales y la integración sur-sur promovidas por la UE. A este respecto, Túnez firmó también un acuerdo de libre comercio con Turquía y con los Estados miembros de la Asociación Europea de Libre Comercio (AELC) que entraron en vigor en 2005, así como un acuerdo bilateral con Libia que entró en vigor en 2002.

Con la puesta en marcha de la PEV en 2004, el marco institucional de la asociación estratégica euro-mediterránea se vio reforzado con la aprobación en 2005 del Plan de Acción de la política de vecindad, cuyo objetivo (reiterado a lo largo de las páginas anteriores) es en términos generales "moving beyond the existing relationship to a significant degree of integration, including offering Tunisia a stake in the internal market and the possibility of participating progressively in key aspects of EU policies and programmes". En el terreno agrícola, asunto espinoso de las relaciones euromediterráneas, se formulaba vagamente la prospectiva de 'extender progresivamente' las relaciones comerciales al sector agrícola y se

\footnotetext{
148 Carafa, Luigi y Korhonen, Kaisa: "European Neighbourhood Policy: A Case Study of Morocco", SSRN Electronic Working Paper Series (27 Abril 2008), pp. 7-8.

${ }_{149}$ EU-Tunisia Euro-Mediterranean Agreement (1998), Official Journal of the European Communities, 30 Marzo 1998, L 097 , pp. 2-183.

${ }^{150}$ Complementado por fondos del Banco Europeo de Inversiones de 1.518 millones de euros para el mismo periodo. Commission of the European Communities (2004c): "European Neighbourhood Policy. Country Report Tunisia \{COM (2004)373 final\}, Commission Staff Working Paper, 12 May 2004, SEC (2004)570, p. 4.
} 
enfatizaba en la necesidad de aplicar las medidas de seguridad alimentaria de la UE con el fin de "improve the health safety of food and facilitate trade in agricultural products with the EU on the basis of reforms and modernisation of the veterinary and plant health sectors". ${ }^{151}$ Las negociaciones en el ámbito agrícola se han demostrado difíciles y por el momento prácticamente todos los productos agrícolas están sujetos a aranceles, exceptuando algunas cuotas para productos específicos como el aceite.

Debemos tener en cuenta que Túnez es "a food-deficit country despite having achieved near self-sufficiency in dairy products, meat, and various vegetables and fruits. Domestic food production is lower than consumption requirements for key staples such as cereals, vegetable oil and sugar", productos que Túnez importa de la UE. Además, el crecimiento económico tunecino es muy sensible a "the cyclical output of agriculture (vulnerable to poor rainfall) and to the rate of expansion in the European Union (EU), the destination of 80\% of Tunisia's exports", lo cual, junto con el aumento de los precios y la situación de inestabilidad, deja a Túnez en una situación muy vulnerable de posible inseguridad alimentaria. ${ }^{152}$

Si bien podemos afirmar que las relaciones económicas en el marco euromediterráneo han tenido un desarrollo considerable, no se consiguió ningún avance en la esfera política (ningún avance impulsado por la UE), y desde el principio la posibilidad de discusión y reforma política se marginó del plan de acción, como queda reflejado en el Country Report al afirmar, respecto al acuerdo de asociación:

"The Agreement also contains provisions which allow political questions (international, domestic, human rights and democracy) and migration issues to be covered. Tunisia has decided to embark on cooperation in the areas of good governance and justice and home affairs on a very gradual basis only". ${ }^{153}$

La ausencia de los elementos de 'buen gobierno' de las relaciones entre Túnez y la UE ha sido objeto de muchas críticas en relación a la colaboración de la UE con gobiernos autoritarios y manifiestan el énfasis de la Unión en la consecución de los objetivos económicos sobre la retórica de los principios y objetivos promulgados en la política europea de vecindad en lo respectivo a avances políticos y sociales:

"The regime is founding its legitimacy on the provision of material goods in a nearly complete absence of political freedoms. The EU is de facto going along with this approach, but by doing so, it is relinquishing any attempt to have a policy beyond a foreign economic policy (...) It is thus striking to see how Tunisia has been able to separate the economic dimension of the ENP from the political one and set the tone of its relationship with the EU,. ${ }^{154}$

Las relaciones de la UE con Túnez se encuentran actualmente en un momento de revisión después de la denominada 'primavera árabe', y todavía está por ver cuál será el compromiso de la UE en la esfera política y social, cuáles serán los incentivos propuestos y las

\footnotetext{
${ }^{151}$ EU/Tunisia Action Plan, p. 2, 3 y 12.

152 Dhur, Agnès (2011): "Secondary data analysis of the food security situation in Tunisia", World Food Programme, Regional Bureau for the Middle East, ODC (April 2011), p.7.

${ }_{153}$ Commission of the European Communities (2004c), op. cit., p. 4.

${ }^{154}$ Bicchi, op. cit., p. 214.
} 
características concretas de la 'asociación privilegiada' acordada en el Consejo de Asociación UE-Túnez en noviembre de 2012 . $^{155}$

\subsubsection{Argelia}

Las relaciones de la UE con Argelia se basan en el Acuerdo de Asociación firmado en 2002 y en vigor desde $2005 .{ }^{156}$ Sin embargo, y a diferencia de Marruecos y Túnez, Argelia no ha negociado la aprobación de un plan de acción en el marco de la política europea de vecindad ni ha formalizado su participación en diversas estructuras e iniciativas en dicho marco, lo cual ha sido un obstáculo evidente para la intensificación de las relaciones con la UE en función del 'principio de diferenciación' de la PEV. En diciembre de 2011 Argelia manifestó su interés en iniciar "exploratory negotiations" para un eventual plan de acción y comenzó un proceso de discusiones informales, pero por el momento las relaciones con la UE se fundamentan principalmente en el Acuerdo de Asociación, aunque Argelia se incluye como país vecino en los documentos de la PEV y se aprobó un 'paquete de medidas' (ENP package) que supliera la ausencia del plan de acción.

Las negociaciones sobre la supresión de aranceles para el establecimiento de una zona de libre comercio de productos manufacturados han sido también difíciles y se ha llegado a un acuerdo en agosto de 2012 sobre la posibilidad de posponer las medidas hasta 2020 para la mayor parte de los productos industriales. En el ámbito de la agricultura los aranceles también persisten excepto en el caso de algunas cuotas preferenciales. ${ }^{157}$

Las razones para la ausencia del plan de acción son varias y de diversa naturaleza. Por un lado, la difícil situación doméstica ha centrado el interés de la agenda política argelina, desviando la atención de las relaciones exteriores con la UE. Además, la entrada en vigor del Acuerdo de Asociación en 2005 alejó el interés de negociar otro acuerdo complementario, y las reformas económicas propugnadas por la PEV hacia una economía de mercado liberal chocan frontalmente con la naturaleza de la economía argelina, dominada por el Estado, lo cual deja entrever la dificultad de poner en marcha un proceso de liberalización y de reforma estructural. ${ }^{158}$

Además, la economía argelina no está diversificada y es dependiente de las exportaciones de gas y petróleo "with 98 \% of EU imports from Algeria in 2011 being energy, Algeria ranked as EU's third largest energy provider in 2011, at a value of $€ 26.8$ billion. Chemicals represented the second most important product group in EU's imports from the country", siendo Argelia el único socio mediterráneo con el que el saldo comercial no es favorable a la UE dada la naturaleza energética de las importaciones. ${ }^{159}$

\footnotetext{
${ }^{155}$ No consideramos pertinente en este análisis evaluar el impacto de la denominada 'Primavera Árabe' en la evolución de las relaciones UE-Túnez y en la implementación de la PEV debido a la falta de distancia respecto a los acontecimientos. A este respecto remitimos al artículo de Martín, Iván: “¿Qué finalidad política para la Política Europea de Vecindad? Sobre los objetivos e instrumentos de la cooperación de la UE con sus países vecinos", Anuario Jurídico y Económico Escurialense, no XLV (2012) pp. 359-388.

${ }^{156}$ EU-Algeria Euro-Mediterranean Agreement (2005), Official Journal of the European Union, 10 octubre 2005, L 265 , pp. 2-228.

157 Algeria Press Service: “Algeria, EU reach tariff dismantling agreement”, 23 agosto 2012, en http://www.aps.dz/Algeria-EU-reach-tariff.html; y Xinhua: “Algeria, EU Agree to Postpone Tariff Dismantling to 2020", 24 agosto 2012, en http://english.cri.cn/6966/2012/08/24/3241s718590.htm

${ }^{158}$ Bicchi, op. cit., p. 217.

${ }^{159}$ Fuente: http://ec.europa.eu/trade/creating-opportunities/bilateral-relations/countries/algeria/.
} 
Los objetivos prioritarios de la UE para Argelia durante el periodo 2007-2013 incluyen la reforma política en los ámbitos de los derechos humanos, del Estado de derecho y del bueno gobierno; reformas en el sistema judicial, la gestión de los flujos migratorios y la lucha contra el crimen organizado entre otros, pero se suaviza la posición al aclarar que el hecho de constituir una prioridad "does not mean that the Commission will intervene in all these areas, merely that they are eligible and that the priorities and implementation schedule can be reviewed". ${ }^{160}$

El caso argelino pone de manifiesto la relevancia estratégica del Mediterráneo para la UE en la cuestión energética, y el papel fundamental que juega en las relaciones económicas. Tanto en el Proceso de Barcelona como en la PEV se otorga un papel central al sector energético, debido a la dependencia de Europa de los suministros provenientes de los países del norte de África, en su doble vertiente de productores o países de tránsito.

La UE progresivamente vincula la energía a la seguridad y en consecuencia la estabilidad de los países del norte de África se muestra como un elemento fundamental para la seguridad energética de Europa. Esta securitización de la energía condiciona la evolución de las relaciones de la UE con Argelia (y euro-mediterráneas en general) y contribuye a que se difuminen las líneas divisorias entre la seguridad interior y exterior, favoreciendo un enfoque europeo en el que la agenda doméstica se superpone a la agenda exterior, y donde la creación de un área de prosperidad queda en un segundo plano:

"En los últimos cinco años se han intensificado las preocupaciones en relación con la dependencia energética. El descenso de la producción en Europa implica que para 2030 será necesario importar hasta un $75 \%$ de nuestro consumo de petróleo y de gas. Éstos provendrán de un número limitado de países, muchos de los cuales afrontan amenazas contra su estabilidad. Por consiguiente, nos enfrentamos a un abanico de retos a la seguridad que exigen la responsabilidad y solidaridad de todos los Estados miembros. Nuestra respuesta deberá consistir en una política energética de la UE que combine las dimensiones exterior e interior". 161

\section{Conclusiones}

En este artículo hemos abordado el proceso de institucionalización de las relaciones euromediterráneas, y de la política en materia de comercio agrícola particularmente, desde el origen de las primeras iniciativas hasta la actual Política Europea de Vecindad, intentando hacer evidente la relación entre las diversas iniciativas europeas y los intereses en el ámbito político, económico y de seguridad.

Podemos concluir afirmando que los marcos de relaciones formalizados para las relaciones exteriores de Europa con la ribera sur del Mediterráneo no se formulan desde preocupaciones ligadas al desarrollo socio-económico de la región, como se manifiesta en la retórica de los documentos, sino que sirven a otros objetivos de naturaleza endógena que explican las motivaciones reales del compromiso europeo respecto a los vecinos

\footnotetext{
${ }^{160}$ Commission of the European Communities (2007): "ENPI: Algeria Strategy Paper 2007-2013 \& National Indicative Programme 2007-2010”, p. 18, en http://www.enpiinfo.eu/library/content/algeria-country-strategy-paper-2007-2013-and-national-indicative-programme-2007$\underline{2010 .}$.

$\frac{2010}{161}$ Consejo Europeo (2008): "Informe sobre la aplicación de la Estrategia Europea de Seguridad - Ofrecer seguridad en un mundo en evolución”, 11 Diciembre 2008, S407/08, p.5.
} 
mediterráneos y que reflejan la aspiración de la proyección del "softpower" europeo hacia sus 'socios' mediterráneos.

Hemos intentado desglosar los intereses europeos subyacentes a la formulación de las políticas euro-mediterráneas, fundamentalmente en el caso del Proceso de Barcelona y de la Política Europea de Vecindad, con un interés particular en las políticas comerciales de productos agrícolas.

En el caso del comercio agrícola, hemos visto cómo la UE ha negociado acuerdos con los países objeto de nuestro interés particular en este texto (Marruecos, Argelia y Túnez) desde la década de los años sesenta, como respuesta a la progresiva integración interna de la Unión y a sus sucesivas ampliaciones.

Sin embargo, desde el origen de las primeras medidas y la firma de los primeros acuerdos, hemos visto las limitaciones de los enfoques europeos y la protección del mercado interno respecto a los productos competitivos de la orilla sur del Mediterráneo, lo cual ha tenido consecuencias económicas y sociales para las poblaciones de dichos países.

Si con el fin de la Guerra Fría se produce un cambio fundamental en las relaciones euromediterráneas con el nacimiento de la Asociación Euro-Mediterránea y la vinculación creciente de los intereses en materia de seguridad y de desarrollo, en el sector agrícola ésta no supuso un cambio sustancial, al continuar la restricción de las importaciones de productos agrícolas de los países del sur. La UE enviaba así un mensaje contradictorio a los socios mediterráneos, promoviendo la eliminación de las barreras y restricciones comerciales que existían en relación a los intercambios de productos manufacturados y al no incluir en los acuerdos el comercio de productos agrícolas. Esta característica constituirá una de las debilidades más flagrantes del Proceso de Barcelona.

De igual forma, la Política Europea de Vecindad, al surgir como un complemento y refuerzo de la AEM, aun aportando importantes innovaciones, mantuvo los principales principios y objetivos de las anteriores aproximaciones y políticas de la UE hacia el Mediterráneo, perpetuando así las limitaciones que plantea el enfoque ligado a los intereses políticos y securitarios europeos.

Además, podemos afirmar que, aunque la UE ofrece relaciones privilegiadas a sus vecinos con la intención de contrarrestar los efectos perniciosos de la ampliación de la Unión, la ausencia del premio de la membresía ha obstaculizado la posibilidad de la UE de proyectar sus normas y valores fundamentales, a pesar de la oferta de un acceso intensificado al mercado interno.

Por otro lado, el énfasis otorgado al principio de diferenciación, según el cual, cada socio puede hacer evolucionar las relaciones con la Unión en un grado diferente en función de su compromiso y esfuerzos nos ha permitido observar diferencias cualitativas y cuantitativas en torno a la situación de los tres países del Magreb estudiados, Marruecos, Túnez y Argelia, lo cual refleja la diferente capacidad de atracción de la UE en contextos políticos y económicos diferentes.

El caso de Marruecos es el más llamativo, y sus progresos se han visto recompensados con el fortalecimiento de sus relaciones bilaterales con la UE hasta el punto de obtener el estatuto avanzado y de firmar en 2012 un acuerdo sobre el comercio de productos agrícolas, de importantes consecuencias para el desarrollo potencial del sector agrícola marroquí. Sin 
embargo, en el caso de Túnez, la situación ha sido muy diferente, no únicamente en el sector agrícola, en el que el país parte de un déficit de producción y es muy vulnerable a una situación de inseguridad alimentaria, sino también en el terreno político, siendo las relaciones entre Túnez y la UE objeto de muchas críticas en relación a la colaboración de la UE con gobiernos autoritarios, desvirtuando así su retórica en lo que respecta los avances políticos y sociales. En relación a Argelia, las relaciones han estado basadas en el sector energético, y las negociaciones en torno al establecimiento de una zona de libre comercio de productos manufacturados han sido difíciles al no existir un plan de acción en el marco de la Política Europea de Vecindad, razón por la cual los aranceles persisten también en el ámbito de la agricultura, excepto en el caso de algunas cuotas preferenciales.

Por último, al difuminarse las líneas divisorias entre las cuestiones de seguridad externa e interna, ha quedado condicionada la evolución de las relaciones euromediterráneas y de la formulación de las distintas políticas desde el final de la Guerra Fría. Los intereses securitarios han impregnado la asociación estratégica y la PEV, generando un proceso asimétrico en el que la UE ve a los vecinos del sur no como socios igualitarios sino como fuente de posibles amenazas a la estabilidad europea, fundamentalmente en el terreno de la inmigración ilegal y del abastecimiento energético. Esta progresiva securitización de las políticas ha impedido el desarrollo del pleno potencial de la política europea de vecindad, al favorecer un enfoque limitado en lugar de una visión reformadora y estratégica hacia la región, que pudiera generar una mayor transformación política y económica.

La política en materia de comercio de alimentos con los países vecinos de la orilla sur del Mediterráneo no se ha formulado desde preocupaciones ligadas a la seguridad alimentaria ni al desarrollo económico de dichos países, sino que han entrado en juego otras prioridades europeas en el ámbito de la seguridad y de la estabilidad, así como de la agenda normativa de la Unión Europea. 